\title{
从交叉学科到学科交叉: 美国案例及启示
}

\author{
王孜丹 ${ }^{1,2}$, 杜鹏 $^{1^{*}}$ ，马新勇 ${ }^{3}$ \\ 1. 中国科学院科技战略咨询研究院, 北京 100190 ; \\ 2. 中国科学院大学, 北京 100049 ; \\ 3. 中国科学院学部工作局, 北京 100190 \\ *联系人, E-mail: dupeng@casipm.ac.cn
}

当前, 新一轮科技革命和产业变革正在孕育兴起, 并深 刻地改变着当前的科研范式以及世界的发展格局. 面对复杂 的现实挑战和多变的发展形势, 交叉融合越来越成为科学研 究的重要领地和解决问题的新模式 ${ }^{[1,2]}$. 2015年9月17日的 Nature杂志开设专栏，详细讨论了“交叉学科研究为什么重 要”[3]; 从诺贝尔奖获得者也可以看出, 物理学、化学以及生 物学和医学之间的界限越来越模糊，它们不仅相互交叉和渗 透，而且逐渐形成了许多界限模糊的连续区间并产生了大量 的科学新生长点 ${ }^{[4]}$.

从广义上讲, 交叉学科指由两门或两门以上不同学科交 叉渗透形成的学科，包括了多学科(multidisciplinary)、交叉 学科(interdisciplinary)以及跨学科(transdisciplinary)的意蕴内 涵. 从狭义上讲, 交叉学科指的是不同学科交叉所形成的新 学科, 它来自被交叉的原有学科, 但又不同于已有学科 ${ }^{[5]}$. 在 飞速发展的科技形势和日益激烈的全球竞争当中，越来越多 的科研发现产生于传统学科的交界处; 越来越多的现实性问 题需要跨越学科界限、集成多方利益相关者的力量来协同 解决. 相应地, 将研究和教育结合起来, 通过交叉学科训练培 养能够以多元创新方式应对科学挑战的科研后备人才也成 为了交叉融合背景下的关键问题. 可以说, 它既是实现科学 知识系统整合的重要基础，又是孕育重大科技创新的现实通 道, 也是新时代复合型人才的培养方式.

尽管发展交叉科学已经成为科技界的共识，但其成长 路径并不顺利. 长期以来, 自然科学、社会科学、人文科学 之间存在着不可逾越的鸿沟 ${ }^{[6]}$. 随着科技界、政府以及科研 管理部门从科学研究、科学政策以及科研管理上对交叉学 科的关注和重视，教育部和国家自然科学基金委员会(以下 简称“基金委”) ${ }^{[7,8]}$ 对各自学科布局的机制和模式开展了相应 的优化与改革尝试. 特别是在 2020 年 8 和 10 月, 全国研究生教 育会议和基金委分别决定新增交叉学科作为我国第 14 个学 科门类 ${ }^{[9]}$ 和新增交叉科学部(http://www.nsfc.gov.cn/publish/ porta10/tab440/info79287.htm), 正式推进交叉学科的发展. 然而, 交叉学科与传统学科存在诸多差异, 发展交叉学科也 面临着许多挑战 ${ }^{[10]}$. 在国家制度层面上确立交叉学科仍然

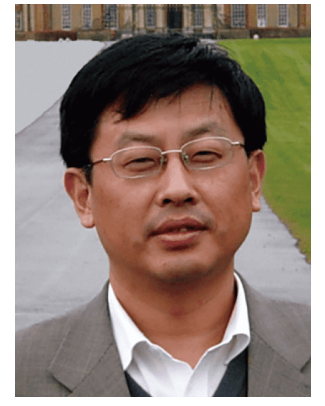

杜鹏中国科学院科技战略咨询研 究院研究员, 中国科学院学部学科研 究支撑中心执行主任. 中国科学学与 科技政策研究会理事, 科学社会学专 委会秘书长，《中国大百科全书》第 三版管理科学与工程卷“一般管理理 论”分支副主编，中国科学技术协会 “世界一流学会建设项目”专家委员 会委员。研究方向为科技政策、科学

技术与社会和学科政策.

属于新生事物, 如何设置组织机构、建立相应机制、培养交 叉科学观念仍然面临很多挑战, 从单独设立交叉学科门类到 学科交叉蓬勃发展并极大提升科研原创力可以说是任重而 道远.

因此，本文以美国国家科学基金会(NSF)和教育部的交 叉学科布局情况为案例, 梳理美国布局交叉学科的制度经验, 对设立交叉学科的作用和逻辑进行归纳总结, 重点关注在新 增交叉学科门类后，应该建立什么样的交叉学科运转机制能 够充分发挥设立交叉学科的作用、进而促进科研原创力的 提升和复合型人才的培养, 为我国下一步完善交叉学科的组 织制度、早日达成学科交叉的建设目标提供参考.

\section{1 交叉学科的布局实践: 美国国家科学基金 会与美国教育部}

交叉学科的设置在学科布局上具有里程碑式的意义. 正 如我们所知, 学科布局具有促进知识生产和促进知识传承的 双重功能. 从国家层面上来讲，各国科学基金和教育部门都 会分别依据自身需求，按照促进知识生产和促进知识传承两 个不同功能对包括交叉学科在内的科学知识进行布局和设 置. 因此，以美国国家科学基金会和美国教育部对交叉学科 的布局为案例，阐述其机制与经验，为我国交叉学科的下一 步发展提供参考. 


\section{1 美国国家科学基金会}

作为国家重要的科研资助机构, 各国基金会的学科布局 不仅承担了资助课题项目的功能，对知识的生产和新学科的 形成也起到一定的引领和带动作用. 因此，在基金会设立专 门处理交叉学科项目的部门对新兴交叉领域的知识生产、 人才成长以及科学共同体的职业生涯发展都很有必要. 而在 交叉学科的组织建设中, 机构职能的设置和处理提案的机制 是两个主要的方面.

\subsection{1 交叉学科的机构设置与职能}

长期以来, NSF对交叉学科的发展都比较重视. 在NSF的 机构, 不仅在整合活动办公室中设置了相关部门负责涉及整 个基金会的、跨越多个科学部的交叉研究, 还分别在生物学 部、工程学部、数学和物理科学学部以及社会、行为和经 济科学部分别下设了科学处来处理与各自学部相关的交叉 学科事项. 具体设置如图1所示.

(i ) 整合活动办公室. 整合活动办公室(Office of Integrative Activities, OIA)隶属NSF主任办公室(Office of the Director, OD), 主要负责交叉学科的活动、领导和协调涉及 整个基金会的事项. OIA下设 4 个职能部门，其中主要负责交 叉学科事务的部门是整合活动科(Integrative Activities Section, IA)和融合加速办公室(Convergence Accelerator Office, CAO).

IA管理并主导NSF范围内主要的交叉学科研究计划. 例 如, 科学技术中心主要研究仪器、中型研究基础设施-1、历 史悠久的黑人大学-卓越研究、日益增长的融合研究和
NSF2026. 另外, 对各学部下设交叉科学处主导的其他交叉学 科资助项目，整合活动科也会积极配合，在评审和资助等方 面共同推进. 整合活动科希望通过制定并协调大规模的跨部 门资助计划, 形成一个强大的研究集团, 超越学科边界, 促进 协作并使用创新方法来实现科学突破.

CAO是NSF于2019年新成立的一个部门，通过多方利益 相关者的合作关系, 集成多学科研究与创新过程, 致力于在 国家具有重大需求的领域开展面向应用的融合研究. 融合加 速办公室资助的研究以基础研究为源头, 多元化的团队成员 采用交叉学科的融合方法，通过固定项目期限和办公室的主 动管理加速可交付成果的实现, 使基础研究朝着有效解决方 案的方向快速发展. 在主题的选择上，办公室定期举办主题 选择研讨会，收集包括企业、学术机构、政府以及非营利组 织等在内的多方利益相关者的意见，在宽泛的主题下确定每 一阶段具体的资助主题，以面向国家需求快速实现经济和社 会利益的相关计划. 融合加速办公室的工作开展情况如表 1 所示.

(ii) 各学部下设的科学处以及配合部门. 在NSF的7个 学部中, 有 4 个学部都在学部内下设了科学处来专门负责交 叉学科的相关事项(图1). 4个科学处跨越了传统的学科界限, 一方面与OIA和其他学部在项目评审、资金分配等方面相互 配合，共同推进全委层面上的交叉学科资助计划; 另一方面 负责本学部内部的交叉研究，与各自学部内的其他科学处合 作, 为学部内交叉学科的项目提供申请和资助上的便利, 并 主导若干横向交叉项目, 突出了学部的整体性. 同时, 作为各 自学部下设的实体机构, 上述科学处还为各自学部的伙伴关

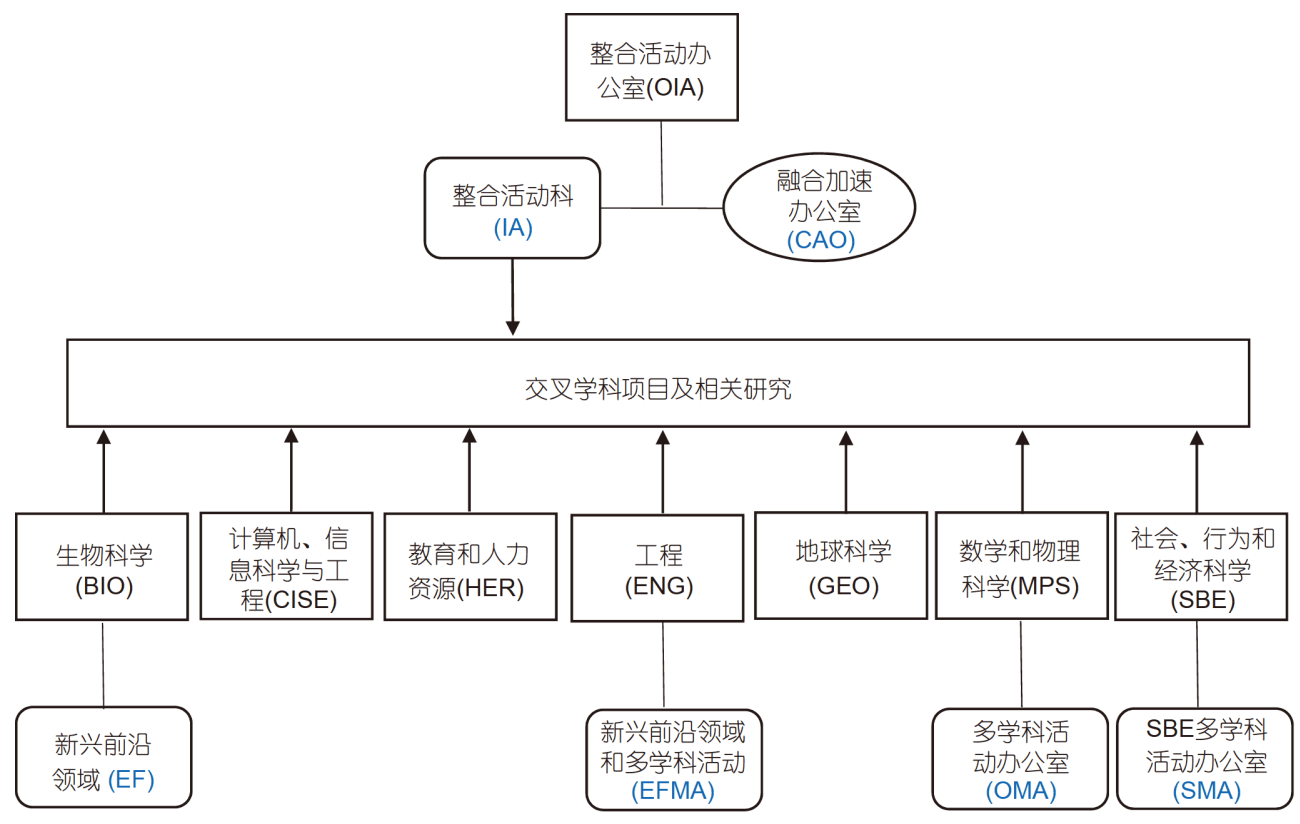

图 1 (网络版彩色)NSF负责交叉学科的机构设置

Figure 1 (Color online) Institutions responsible for interdisciplinary in NSF 
表 1 融合加速办公室的资助情况 ${ }^{\text {a) }}$

Table 1 Funding status in CAO

\begin{tabular}{cccc}
\hline 年份 & 阶段 & 项目代码 & 资助主题 \\
\hline \multirow{2}{*}{2019} & 试验阶段一 & $19-050$ & Track A: 利用数据革命 \\
& 试验阶段二 & $20-555$ & $\begin{array}{c}\text { Track B: 在人-技前沿工 } \\
\text { 作的未来 }\end{array}$ \\
2020 & 第一阶段和第二阶段 & $20-565$ & $\begin{array}{c}\text { Track C: 量子技术 } \\
\text { Track D: 人工智能 }\end{array}$ \\
\hline
\end{tabular}

a) 资料来源: https://www.nsf.gov/od/oia/convergence-accelerator/

系(如与行业、国家实验室、州和地方政府以及国际组织)提 供了一个协同中心，便于产学研之间的沟通与整合，为多学 科环境下的协同合作与教育模式提供新方案.

除此之外, 计算机与信息科学与工程科学部、教育和人 力资源科学部以及地球科学部虽然没有专设负责交叉学科 的科学处，但是在实际的项目接收、评审以及资金分配的过 程中, 这 3 个科学部也会与上述部门相互配合, 形成了一个跨 部门的NSF交叉学科项目主管协作团队，共同完成交叉学科 的项目提案.

总的来说，NSF在战略上对交叉学科的发展十分重视, 具体而细致的机构设置与职能分配保证了交叉学科研究拥 有自由充裕的发展空间. 一方面通过整合活动科、学部下设 科学处以及其他部门的配合, 形成跨部门的NSF计划主任协 作机制，共同接收、审议和资助交叉学科的项目提案，见 图2; 另一方面, 融合加速办公室的设立, 有利于加速开展可 以及时回应国家需求的交叉融合研究, 大大拉近了产学研之 间的合作伙伴关系. 上述布局设置使NSF在交叉学科的发展 上既具备应对长期复杂挑战的能力，又有及时支持重要新兴 领域的敏捷性, 保证了交叉学科得以充分发展.

\subsection{2 交叉学科的提案机制}

在处理交叉学科的组织机制上，最重要的部分就是应当 如何提交和审查交叉学科的项目提案，以保证所有有关交叉 学科的想法都能不被忽略. 在向NSF提交交叉学科的项目提 案时，可以根据NSF已有的资助项目确定项目官员，也可通 过联系相应学部或办公室等其他方式确定项目官员. 具体的 提案程序如图3所示.

根据交叉学科项目提案的处理程序, 可以看出各学部和 办公室的项目官员已经形成了十分密切的沟通网络，在处理 提案的过程中并不只筛选符合本部门要求的提案，还会对适 合其他学部的提案进行推荐并对跨学部的交叉提案进行联 合讨论. 大大减少了交叉学科的项目提案被忽略的可能性.

\section{2 美国教育部}

为了便于组织、收录和公布全国中学后教育机构开设 的学科专业, 进行分类并赋予代码, 提供信息服务, 美国国家 教育统计中心(National Center for Education Statistics, NCES)

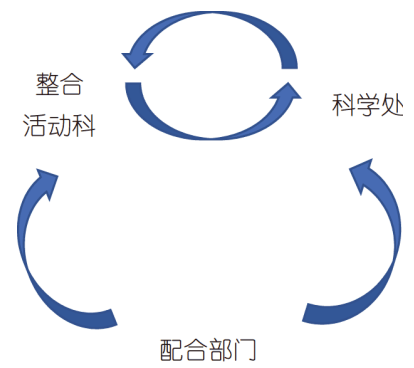

图 2 (网络版彩色)跨部门的NSF交叉学科协作机制

Figure 2 (Color online) Interdisciplinary collaboration mechanism across departments in NSF

于1980年研究开发了“Classification of Instructional Programs”(CIP), 并由美国教育部(U.S. Department of Education, USDE)发布 ${ }^{[11]}$. 考虑到CIP涵盖所有中学后教育, 本文将其译 为“学科专业分类目录”. CIP的构成为 2 位数、 4 位数与 6 位数 代码 3 个层次，分别对应我国学科专业目录中的学科门类、 一级学科和二级学科.

\subsection{1 交叉学科的代码变化}

1980年以来, NCES先后发布了CIP1985、1990、2000、 2010 和2020五个修订版. 而早在1985年的修订版中就已经为 交叉学科专门设置了“30多学科/交叉学科”(multi-/interdisciplinary studies)学科专业群. 图4展示了1985年以来CIP在“30 多学科/交叉学科”学科专业群上的动态变化.

整体来看, 从1985 2020年, 交叉学科的四位代码和六位 代码数量都在不断上升，基本保持了每 10 年净增约 10 个代码 的速度. 尤其是在近 10 年, 代码增速最快, 代码数量翻倍, 发 展势头十分迅猛.

具体来看，CIP中交叉学科代码历经 30 余年的发展及演 变, 呈现出以下两个基本趋势与主要特点: 第一, 从代码内容 上看，学科领域间的交叉融合程度逐渐加深．在1985年首次 设立交叉学科代码时, 仅有如30.0301 工程及其他学科(engineering \& other discipline)、30.0401 人文与社会科学(humanities \& social science)等领域特征鲜明, 交叉程度相对较浅的 交叉学科; 随着不同领域和不同层次间科学知识交叉融合的 不断深人，在2020修订版中，新增了许多大跨度交叉和问题 导向鲜明的研究领域, 比如30.39 经济学与计算机科学 (economics and computer science)、30.50 数学与大气/海洋科学 (mathematics and atmospheric/oceanic science)、30.37 人体健 康设计(design for human health)等. CIP-2020已经基本形成了 跨越自然与人文、全面覆盖各科学领域、代码分类细致具 体、问题导向凸显的交叉学科代码体系. 第二，从代码结构 上看，交叉学科逐渐形成了从孵化、新增、发展到建制化移 出的完整代码链条. 30.9999 多学科/交叉学科(其他)(multi/interdisciplinary studies，other)和30.0000 多学科/交叉学科(综 合)(multi-/interdisciplinary studies, general)为新生交叉科学知 识和交叉学科的融合发展提供了容身之所，同时也为新增代 


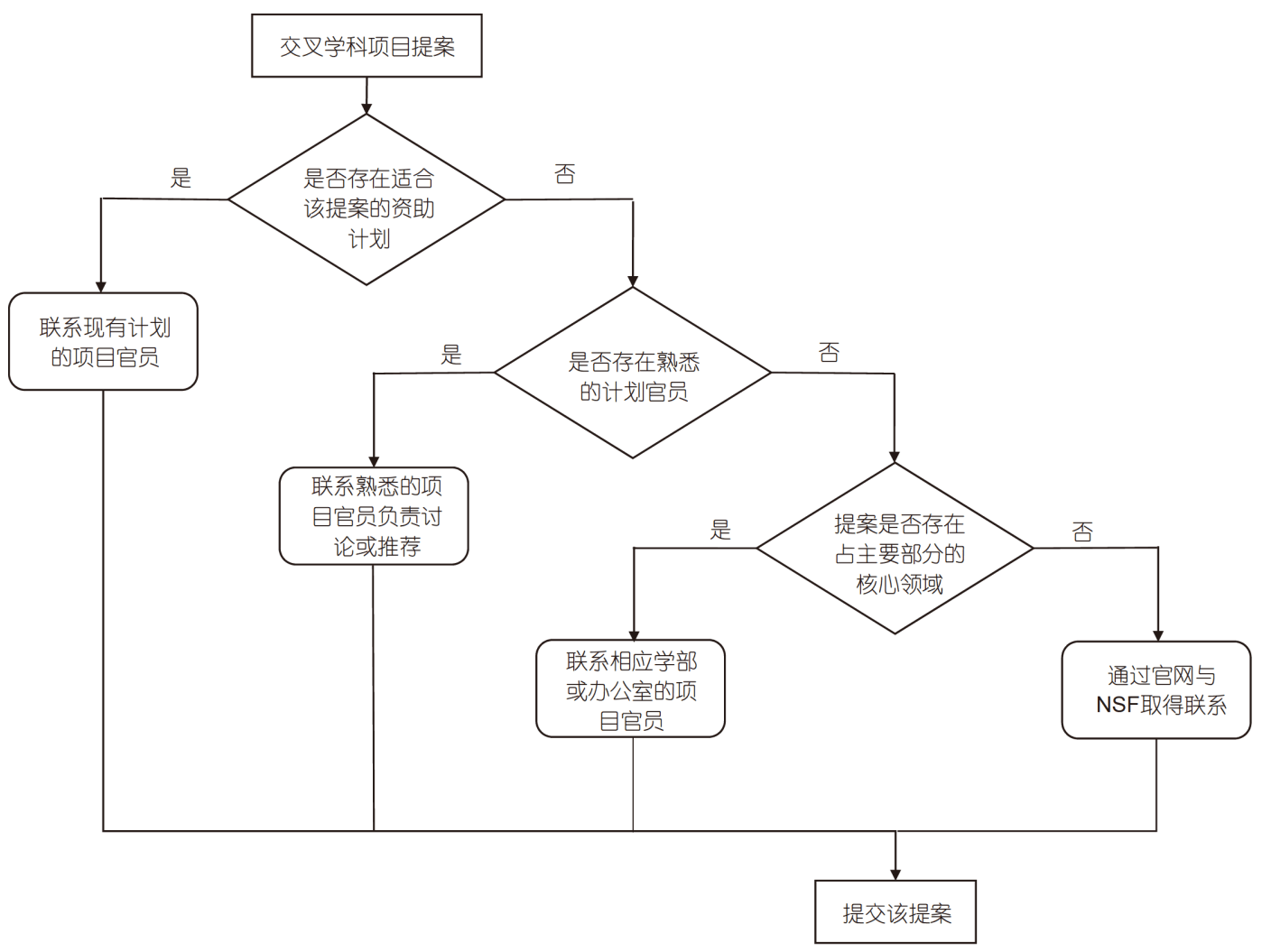

图 3 交叉学科项目提案的处理程序

Figure 3 Processing procedures about interdisciplinary project

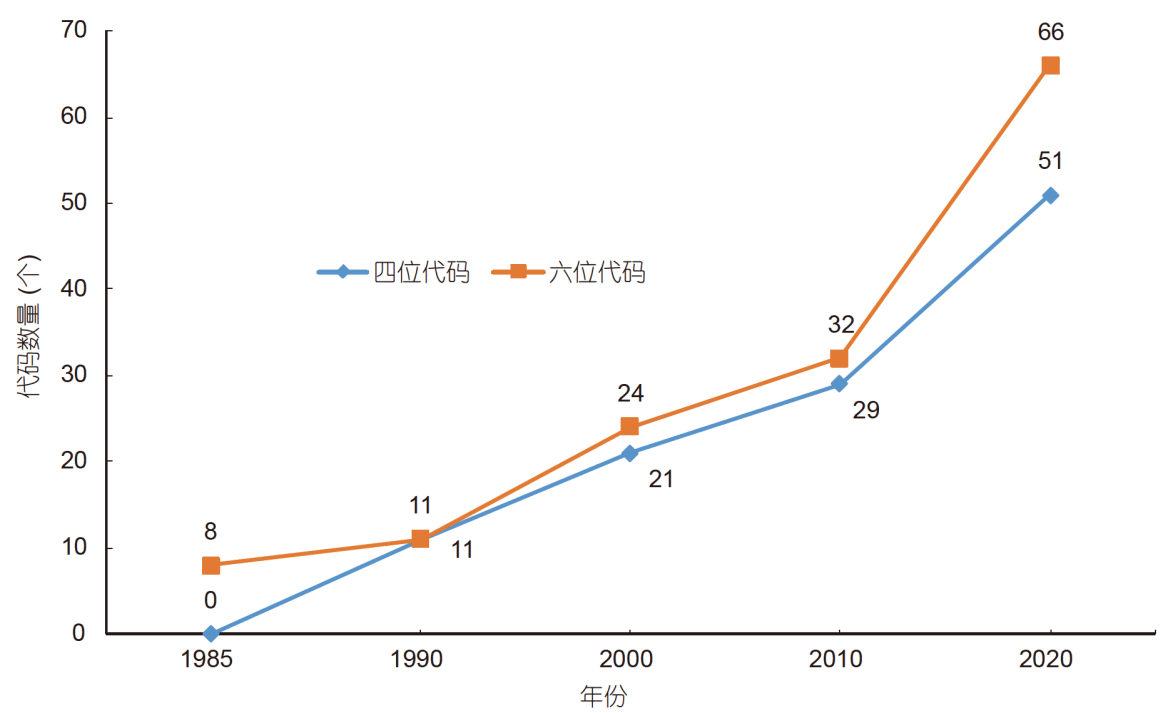

图 4 (网络版彩色)1985 2020年“30多学科/交叉学科”的代码变化. 数据来源: https://nces.ed.gov/pubsearch

Figure 4 (Color online) Code changes about "30 multi-/interdisciplinary studies" in 1985-2020. Data are from https://nces.ed.gov/pubsearch

码提供了备选. 此外, CIP对交叉学科中发展态势良好的领域 则建立了完整的建制化移出机制，30.0201 临床精神关怀 (clinical pastoral care)、30.0301 工程及其他学科(engineering
\& other discipline)、30.0401 人文与社会科学(humanities \& social science)、30.0701 妇女研究(women's studies)以及 30.2401 神经科学(neuroscience) 5 个学科发展成熟, 其六位代 
码分别于1990和2010年移位至各自相应的学科专业群下. 可 以说，CIP对交叉学科代码的设置为新兴领域提供了完整的 全链条发展通道, 为交叉学科提供了良好的制度体系和广阔 的发展空间.

\subsection{2 交叉学科的变动准则}

交叉学科的代码变动是修订的重要部分. 在修订的机制 上, NCES采用了自上而下与自下而上相结合的修订方式, 将 广泛调研与主动申请相结合. 一方面通过中学后教育集成数 据系统的学业调查(The Integrated Postsecondary Education Data System Completions Survey, IPEDS)资料考察所有6位 数代码中以“99其他类”结尾的学科专业，寻找有可能成为新 交叉学科的对象, 并对其进行网上研究和技术审查; 另一方 面, 任何一所高校都能通过IPEDS提出新增学科专业的申请. NCES认真研究每一项申请, 核对所申请的学科专业是否满 足设置的条件要求. 在这种情况下, 一般要求至少要有 5 所高 等教育机构(指的是CIP2020中规定的第IV类中学后教育机 构)符合设置该学科专业的条件, 才能考虑予以增加.

根据CIP-2020修订版的要求, CIP的学科专业需满足以 下4个设置准则: (1) 教学课程必须经由第IV类高等教育机构 或其他得到认可的教育机构提供或赞助; (2) 办学者必须通 过教育部认可的机构认证或预认证, 被认定有资格成为第IV 类高等教育机构, 并获得相应执照或法律授权可以在所在地 办学; (3) 该学科专业必须包含一门以上独立课程或学习经 验, 不能是不相关的课程或经验的随意集成; (4) 必须有一套 由高校或其他办学机构定义的结构化学习经验，符合正式授 予学位、正式学历或一定认可形式的完成学业标准.

对于代码的删除，CIP-2020并无明确要求．事实上在交 叉学科代码的实际变动中并未出现代码被完全删除的情况, 所有变更都是根据实际发展情况移位至其他学科专业群下.

\subsection{3 交叉学科的学位授予}

美国的学位授予是依据CIP中6位数的学科代码, 也就是 具体的学科专业(specific instructional program)作为高校进行 学位授予、上报学位数量的类别依据. 对交叉学科内已有的 学科专业, 按照相应的6位数学科代码对毕业生进行学位授 予; 对于高校自主开设的、现存代码中没有的学科专业，则 可归人30.9999 多学科/交叉学科(其他)(multi/interdisciplinary studies, other)对毕业生进行学位授予.

总体来看, CIP自上而下调研与自下而上申请相结合的 修订方式给交叉学科的人选和增设提供了较大空间. 且CIP2020 修订版中新学科专业的设置准则较CIP-2000修订版相比 更加宽泛，不再作出“近 3 年内至少有 3 个州的 10 个以上高等 教育机构授予至少 30 个该学科专业的学位 ${ }^{[12] ”}$ ”的限定, 这对 交叉学科代码数量的增长起到了较大的促进作用.

\section{3 小结}

根据上述案例可以看出NSF在交叉学科的分工上各部
门协同合作，共同推进交叉学科项目的顺利进行，在全基金 会内形成了比较全面的跨部门交叉学科协作机制. 在交叉学 科项目提案的处理程序上，项目官员间形成了共同讨论或相 互推荐的密切沟通网络, 最大限度地保证了创新性的想法不 会被忽略. 在CIP代码中, “30多学科/交叉学科”的代码数量增 长迅速，代码调整也比较频繁. 自上而下与自下而上相结合 的修订方式对全面网罗新兴交叉领域很有帮助，而根据代码 变动准则以及交叉学科实际情况对发展成熟的交叉学科进 行调整和移位的工作也已经制度化，基本形成了交叉学科代 码从建立到建制的完整发展链条, 全面而详细的代码布局十 分有利于新兴领域的孵化与培育. 另外，基于六位代码的学 位授予制度也为学位授予工作和考察人才的具体研究方向 提供了便利.

总的来说, 美国已经形成了一套较为成熟的交叉学科发 展模式. 这一系列的交叉学科组织制度建设为学科真正的交 叉融合提供了制度便利和发展空间，为从交叉学科的布局设 立到学科交叉的真正实现提供了快速通道.

\section{2 交叉学科的逻辑蕴涵}

\section{1 平衡交叉学科在动态发展与制度保障之间的 张力}

任何学科的发展都是从临时到相对稳定、从不成熟到 成熟的动态过程, 交叉学科在其中起到了孵化培育的作用. 不论学科处于何种发展阶段, 这些因学科分化与科学的发展 需要而建立起来的学术基层组织和结构始终都应存在并为 学科发展提供方便 ${ }^{[13]}$. 在这个过程中, 美国通过设立临时办 公室和“99其他类”代码等方式为动态发展的交叉学科设置了 细致而全面的归属. 例如, 1998年, 极地项目作为重点支持的 新兴交叉学科领域. NSF曾设立极地计划办公室专门致力于 极地地区的创新性科学研究、工程和教育，并与当地居民以 及政府开展密切合作，促进对极地系统及其全球相互作用的 基本发现和了解. 经过 20 余年的发展和数次机构调整，极地 项目这一交叉领域发展成熟并最终于2019年被纳人地球科 学部. 类似地, 环境研究与教育是NSF于 2019 年增设的临时办 公室, 通过跨学科的融合研究和跨部门的方法集成, 发现并 且解决具有社会实用性的基础环境研究和教育问题. 在CIP 中, 除了专设交叉学科门类之外，另设的“99其他类”代码不 但可以容纳新兴的探索性领域，还为交叉学科代码的动态调 整提供了潜在的发展对象. 上述措施保证了任何发展阶段的 交叉学科都拥有相应的归属, 同时也避免了参与者在项目申 请、职称评定、人才培养等资源分配问题上因制度存量不 足而存在劣势的可能性，较好地平衡了交叉学科在动态发展 与制度保障之间存在的张力.

从学科布局的角度来说, 交叉学科的设立是为新知识提 供一种制度化的发展平台和合法身份，用以引导促进某种群 
体认知的形成以配合领域内正在发展的科学知识, 目的是给 知识生产、科研管理乃至成果应用提供某种方便, 属于引导 科技发展的指向性制度政策和布局体系. 因此，知识即已经 被生产, 就需要一定的组织制度与之匹配, 在这个动态的过 程当中, 它们相互辅助、互为表里、共同成长.

\section{2 加强学科协同促进跨学科合作}

学科边界是学术活动的分工和知识分化的必然结果. 跨 学科研究一般有其超越特定学科的共同对象、目标和问题, 但在具体研究过程中, 各个学科所承担的部分, 往往是通向 共同核心问题解决链条中的一个个节点, 这些节点彼此存在 有机且密切的联系. 故而, 合作参与也的确会为不同学科知 识生产提供新的发展与突破机遇. 但是, 跨学科研究不仅依 旧没有消解原有学科, 而且新的理论发现与突破反而有可能 为学科进一步裂变创造条件 ${ }^{[14]}$. 这也是设立交叉学科的逻辑 起点. 从本质上讲, NSF设立交叉学科是在承认学科边界的基 础上，促进跨学科协同合作，以碰撞、交融、衍生、裂变的 不同方式, 构成人类科学前沿的不断拓展.

人类知识增长与学科分门别类化是内外不同逻辑与力 量交织、共同作用的动态过程，不存在一个完全的稳定态. 正是基于这种潜在或显在的变动不居格局，美国的学科分类 目录, 并非一种自上而下的建构, 而是对动态性经验现实的 概括归纳，故更具开放性和调整的灵活性. 由于主要服务于 政府的统计目的, 学科目录不作为干预和约束高校学科专业 自主设置的依据 ${ }^{[15]}$. 与此相比, 我国的学科目录略显刚性, 这 也对学科协同提出了一些制度体系的保障要求和难题，比如 研究资助与人才培养与教育的关系问题，又如教师联合聘 任、联聘教师的绩效评价、学科资源分配机制等一些具体 问题.

\section{3 培养多元主体协作观念有利于问题导向研究}

与传统的学科发展和统摄问题的方式不同, 交叉学科更 多的是围绕重大研究问题或目标，在应用情景中组织起来. 这不仅出于发展学科认知的需要, 更多地体现出交叉学科组 织模式中带有的实用性目的和解决问题的责任，属于问题导 向的研究 ${ }^{[16]}$. 这就意味着要完成复杂的现实问题, 需要多学 科的集体参与和多部门协商解决, 单凭某一学科的力量是很 难完成的. 不论是在交叉学科的机构设置上还是项目提案的 处理程序上, NSF都执行跨部门的交叉学科协作机制, 以解决 问题为原则进行了多部门的协同合作，体现了交叉科学观下 形成的一种更广泛意义上的多元群体认同. 类似地, CIP中 “00综合类”代码的设置也体现了学科交叉融通的综合化发展 趋势. 而 CAO 就是NSF为加速解决相应研究问题而专设的办 公室. 融合加速办公室资助的每一个项目都有十分明确的资 助主题和完成时间, 充分体现了采用学科交叉和解决国家需 求的鲜明特征.
在整个解决问题的过程中, 多学科专家、政府、企业、 社会组织乃至公众等多方利益相关者共同参与了交叉学科 的组织建制，不同学科、不同领域的科学知识、理论方法与 组织模式交叉碰撞、相互耦合, 而由此形成的多元异质的功 能性沟通网络和综合性解决方案改变了传统的科学观念, 逐 渐形成了新的交叉科学观. 在交叉科学观念下, 学科相互融 通并以一种问题导向的组织模式继续发展.

\section{3 建议与展望}

布局交叉学科在本质上是促进学科协同, 为新的前沿交 叉领域知识生产搭建一个动态的生长路径和发展通道. 通过 培养学科交叉的意识、探索问题导向的科研模式、建立符 合交叉研究特点和规律的组织机制，形成学科交叉融通发展 的状态，进而促进科学技术的高质量发展. 因此，中国对交叉 学科的布局与建设, 应从以下几个方面进行.

\section{1 前瞻布局, 培养学科交叉意识}

从布局交叉学科到形成学科交叉, 是一个比较漫长的过 程. 不论是学科门类内部交叉, 还是跨学科门类的交叉, 都应 该前瞻性地培养学生运用多领域知识解决问题的意识和能 力. 鉴于《普通高等学校本科专业目录》和《授予博士、硕 士学位和培养研究生的学科、专业目录》作为高等教育学 科布局的继承性和连贯性, 有必要对本科阶段的学科专业目 录进行相应调整. 增设交叉学科为新的学科门类或一级代码, 甚至有必要将交叉学科的思想延伸到义务教育阶段的科学 教育当中. 美国在STEM教育中对交叉学科的重视, 以及包含 科学和工程实践、交叉学科概念和核心概念在内的新一代 科学教育标准的提出 ${ }^{[14]}$, 为我们提供了很好的示范. 事实上, 鼓励学生参与交叉学科研究活动, 并不真正要求他们产出学 术成果, 关键在于领悟不同学科知识相互启发和促进的过程, 培养学科交叉的意识和能力, 进而加深对知识创新本质的 理解.

\section{2 问题导向, 从不同领域凝练交叉问题}

在CIP-2020的版本中, 新增了许多跨越自然科学和社会 科学的大尺度交叉科学问题和以解决问题为中心的研究领 域，使得交叉学科逐渐形成了更加细致具体的代码体系和更 加完善的学科布局. 因此国内在交叉学科的改革和优化工作 中要更加突出问题导向，引导和鼓励科研人员在解决现实问 题的过程中进一步凝练交叉科学问题; 并以实质性交叉为原 则, 聚焦不同知识范畴中的共性原理和共同科学问题. 坚持 探索新科学研究范式, 努力推动新兴交叉领域发展, 早日取 得重大原创性突破.

\section{3 柔性管理, 给予交叉学科足够的发展空间}

作为新的学科门类, 交叉学科与传统学科差异较大, 给 
学科布局的管理也提出了新的挑战. 鉴于交叉学科的动态生 长性, 不论是学科专业代码还是资助机构的学科布局, 都应 建立较宽松的交叉学科纳人准则和较频繁的交叉学科调整 机制，有利于及时把握交叉学科的实际发展情况，并根据情 况对学科布局作出调整. 同时, 对一些重点领域, 可给予更灵 活的制度支持和更充分的发展时间. 总的来说，在交叉学科 的宏观布局和制度建设上国内起步较晚、缺乏经验，需要更 多的自由和空间根据交叉学科的实际发展情况进行相应的 探索与实践. 因此, 对交叉学科的建设需要根据学科交叉程 度以及现实情况，采取不同的组织制度和发展模式，对其进 行柔性管理.

\section{4 完善有利于交叉学科发展的组织机制建设}

对中国来说，交叉学科的学科门类刚刚得以布局，未来 还有许多组织机制上的具体问题需要完善和解决. 交叉学科
的组织机制既要有利于学科交叉融通的发展路径，又要面向 国家需求和成果转化. 另外, 各个学部内的交叉科学处也要 逐步设立起来，以便于处理科学部内部的交叉学科事项，并 对跨学部的交叉学科形成辅助和配合功能. 在建立并完善交 叉学科门类的组织机制上, 把握其“创新灵活性”和“科学规范 性”的协调与统一 ${ }^{[17]}$, 保持大学学术组织结构的稳定性与适 应性 ${ }^{[18]}$, 是交叉学科门类设置的核心问题.

随着教育部和基金委先后对交叉学科门类进行增设与 布局，中国的交叉学科建设逐渐步人正轨，但这只是学科交 叉融通的起点, 还需要继续探索. 尽管美国在交叉学科方面 有许多经验值得我们学习和借鉴，但是中美之间在科技、 教育以及相应制度上仍然存在一些现实情况不尽相同，因 此我们侧重于交叉学科发展经验背后的逻辑探寻和交叉科 学观念的培养，对于某些具体的做法不必盲目效仿、一概 而论.

致谢＼cjkstart感谢中国科学院学部学科发展战略研究项目(XK2019HXC001)、国家自然科学基金委员会应急管理项目(L1924031)和 国家自然科学基金委员会专项(L2024001)资助.

\section{推葆阅读文献}

1 Wang Z D, Zhao C, Zhang L Q, et al. The policy demand and path choice of discipline layout reform of scientific fund in the new era (in Chinese). Bull Natl Nat Sci Found Chin, 2019, 33: 440-445 [王孜丹, 赵超, 张理茜, 等. 优化自然科学基金学科布局的改革逻辑与路径选择. 中国科学 基金, 2019, 33: 440-445]

2 Du P, Wang Z D, Cao Q. Some trends and enlightenment of world science development (in Chinese). Bull Chin Acad Sci, 2020, (5): 555-563 [杜 鹏, 王孜丹, 曹芹. 世界科学发展的若干趋势及启示. 中国科学院院刊, 2020, (5): 555-563]

3 Brown R. Why interdisciplinary research matters. Nature, 2015, 525: 305

4 Liu Z L, Zhao X C. Interdisciplinary research: The driving force of scientific originality-Take the centurial Nobel Prize in physiology and medicine as an example (in Chinese). Stud Philos Sci Technol, 2005, (6): 107-111 [刘仲林, 赵晓春. 跨学科研究: 科学原创性成果的动力之 源一以百年诺贝尔生理学或医学奖获奖成果为例. 科学技术与辩证法, 2005, (6): 107-111]

5 Ke H Q. Transdisciplinary or interdisciplinary (in Chinese)? University(Academic), 2010, (10): 90-95 [柯华庆. 跨学科还是交叉学科? 大学(研 究版), 2010, (10): 90-95]

6 Lu Y X. The significance of interdisciplinarity and interdisciplinary science (in Chinese). Bull Chin Acad Sci, 2005, (1): 58-60 [路甬祥. 学科交叉 与交叉科学的意义. 中国科学院院刊, 2005, (1): 58-60]

7 Zhou Z H, Zhao W J. Funding system reform for excellence in science: An interview with Jinghai Li, the President of NSFC (in Chinese). Bull Natl Nat Sci Found Chin, 2019, 33: 1-4 [周忠和, 赵维杰. 以基金改革追求卓越科学: 专访国家自然科学基金委员会主任李静海院士. 中国科学基 金, 2019, 33: 1-4]

8 Zhang G J, Fu X F, Zheng Q Y, et al. Chemical sciences transformation in China-Review of the 13th Five-Year Plan of Department of Chemical Sciences, NSFC (in Chinese). Sci Sin Chim, 2020, 50: 681-686 [张国俊, 付雪峰, 郑企雨, 等. 转型中的中国化学一一基金委化学部“十三五” 规划实施纪行. 中国科学: 化学, 2020, 50: 681-686]

9 Lu X D. The time has come to add interdisciplinary summary groups (in Chinese). Chinese Science News, 2020-08-18 [卢晓东. 增设多学科交叉 门类时机已至. 中国科学报, 2020-08-18]

10 Liu Z L. The classifying mode and management meditation about interdisciplinary subjects (in Chinese). Stud Sci Sci, 2003, (6): 561-566 [刘仲 林. 交叉学科分类模式与管理沉思. 科学学研究, 2003, (6): 561-566]

11 National Center for Education Statistics. Classification of Instructional Programs: 2020 Edition. Washington DC: U.S. Department of Education, 2020

12 National Center for Education Statistics. Classification of Instructional Programs: 2000 Edition. Washington DC: U.S. Department of Education, 2002 
13 Fang W, Han S F, Cai S S, et al. Pen notes on the construction of disciplinary institution (in Chinese). Soc Sci China, 2002, (3): 74-91, 206 [方文, 韩水法, 蔡曙山, 等. 学科制度建设笔谈. 中国社会科学, 2002, (3): 74-91, 206]

14 Gao H B, Ju S T. Benchmark of civic scientific literacy: The inspiration of international achievements and the exploration in China (in Chinese). Chin Sci Bull, 2016, 61: 1847-1855 [高宏斌, 鞠思婷. 公民科学素质基准的建立: 国际的启示与我国的探索. 科学通报, 2016, 61: 1847-1855]

15 Yan G C. Academic discipline's connotation, its mechanism and basis for classification (in Chinese). Univ Discip, 2020, 1: 58-71 [阎光才. 学科的 内涵、分类机制及其依据. 大学与学科, 2020, 1: 58-71]

16 Hessels L K, van Lente H. Re-thinking new knowledge production: A literature review and a research agenda. Res Policy, 2008, (37): 740-760

17 Liu Z L, Cheng Y. Research on the setting of interdisciplinary (in Chinese). Acad Degrees Grad Educ, 2008, (6): 44-48 [刘仲林, 程妍. “交叉学 科”学科门类设置研究. 学位与研究生教育, 2008, (6): 44-48]

18 Gao S. Stability and adaptability of the academic organizational structure in research universities (in Chinese). Univ Discip, 2020, (1): 22-24 [高 松. 研究型大学学术组织结构的稳定性与适应性. 大学与学科, 2020, (1): 22-24] 


\title{
From interdisciplinary to interdisciplinarity: American cases and implications
}

\author{
Zidan Wang ${ }^{1,2}$, Peng $\mathrm{Du}^{{ }^{*}}$ \& Xinyong $\mathrm{Ma}^{3}$ \\ ${ }^{1}$ Institutes of Science and Development, Chinese Academy of Sciences, Beijing 100190, China; \\ ${ }^{2}$ University of Chinese Academy of Sciences, Beijing 100049, China; \\ ${ }^{3}$ Bureau of Academic Divisionst, Chinese Academy of Sciences, Beijing 100190, China \\ * Corresponding author, E-mail: dupeng@casipm.ac.cn
}

At present, under the complex background of scientific research paradigm change and fierce global competition, interdisciplinary has attracted more and more attention. In 2020, China's education department and scientific research funding department have added interdisciplinary in their respective discipline layout, which is of landmark significance for China's scientific development. However, we still lack experience in establishing and operating the system of interdisciplinary in the future. The United States has a relatively advanced system and referable experience in the development of interdisciplinary, so this paper takes the interdisciplinary layout of American National Science Foundation and American Department of Education as cases to organize the institutional setup, functions and proposal mechanism of interdisciplinary in American National Science Foundation, and to summarize the code changes, change criteria and degree awarding of interdisciplinary in Classification of Instructional Programs. From the perspective of the development logic and evolutionary connotation of interdisciplinary: (1) There is tension between the dynamic development and the institutional guarantee about interdisciplinary; (2) strengthening disciplinary collaboration can promote interdisciplinary cooperation; (3) cultivating the concept of multi-agent cooperation is beneficial to problem-oriented research; they are the logical implication of interdisciplinary. Finally, some suggestions are put forward for the future development of interdisciplinary in China. (1) To cultivate interdisciplinary ideas through forward-looking layout; (2) to solve practical problems by summarizing interdisciplinary scientific questions from different fields; (3) to give enough space for development through flexible management; (4) to improve the organizational mechanisms that are conducive to the development of interdisciplinary. They will be helpful for China to gradually move from interdisciplinary to interdisciplinarity.

interdisciplinary, discipline layout, problem oriented, National Natural Science Foundation of China, discipline code

doi: 10.1360/TB-2020-1450 